\title{
A 6-18 GHz 5-Bit Phase Shifter MMIC Using Series/Parallel LC Circuit
}

\author{
Kenichi MIYAGUCHI, Morishige HIEDA, Yukinobu TARUI*, Mikio HATAMOTO*, \\ Koh KANAYA**, Michiaki KASAHARA and Tadashi TAKAGI \\ Information Technology R\&D Center, Mitsubishi Electric Corp. \\ 5-1-1 Ofuna, Kamakura-city, Kanagawa 247-8501, Japan \\ E-mail: k-miya@isl.melco.co.jp \\ *Kamakura Works, Mitsubishi Electric Corp. \\ ${ }^{* *}$ High Frequency \& Optical Semiconductor Division, Mitsubishi Electric Corp.
}

\begin{abstract}
A high performance 6-18 GHz 5-bit reflection type phase shifter MMIC is presented. It employs a novel ultra-broad-band circuit design technique utilizing a series/parallel LC circuit for an $180^{\circ}$ phase shift over all frequencies. The fabricated phase shifter MMIC with SPDT switch suitable for T/R modules has demonstrated a typical insertion loss of $9.4 \mathrm{~dB}+/-1.4 \mathrm{~dB}$, a maximum RMS amplitude error of $0.33 \mathrm{~dB}$ and a maximum RMS phase error of $7^{\circ}$ over entire operating bandwidths.
\end{abstract}

\section{INTRODUCTION}

Broad-band phase shifters have been required in wideband phased array systems for electronic beam steering. A reflection type phase shifter is able to operate in a relatively wide frequency range over other circuit designs [1]-[3]. However, the chip size is tending to be large mainly due to $3-\mathrm{dB}$ hybrid couplers used in it. To minimizing the size, the high-pass/low-pass switched structure was applied to broad-band phase shifter circuit designs at the expense of the operating bandwidths [4][5]. Another approach to broad-band phase shift characteristics is to employ the analog phase shift circuit design techniques [6][7]. Though the size of the analog phase shifter is small, it cannot achieve a flat phase shift versus frequency over the control voltage.

This paper describes a broad-band reflection type 5-bit phase shifter with the accomplishment of a high performance and a small size. A circuit for each bit of the phase shifter is composed of a broad-band 3-dB Lange coupler and a pair of reflective terminating circuits. With a novel ultra-broad-band circuit design technique utilizing series/parallel LC circuit, an exact phase shift of $180^{\circ}$ can be achieved theoretically for all frequencies [8]. The 6$18 \mathrm{GHz} 5$-bit phase shifter MMIC with single pole double throw (SPDT) switch has been designed and fabricated. The phase shifter MMIC with SPDT switch has achieved a typical insertion loss of $9.4 \mathrm{~dB}+/-1.4 \mathrm{~dB}$, a maximum RMS amplitude error of $0.33 \mathrm{~dB}$, and a maximum RMS phase error of $7^{\circ}$ over the operating frequency band. This excellent performance is suitable for wideband phased array applications.

\section{DESIGN}

The schematic diagram of the proposed 5-bit phase shifter MMIC with SPDT switch suitable for transmit/receive $(T / R)$ modules is illustrated in Figure 1. The MMIC chip is composed of a phase shifter section and a series-shunt FET SPDT switch section. The phase shift circuits design of the 5-bit phase shifter is based on the reflection type topology consisting of a 3-dB Lange coupler and a pair of reflective terminating circuits with switching FETs. The fabricated 5-bit phase shifter MMIC with SPDT switch is shown in Figure 2. The IC has been fabricated by using $0.5 \mu \mathrm{m}$ p-HEMT technology. The total chip size is $4.27 \mathrm{~mm} \times 3.17 \mathrm{~mm}$ including the 5-bit phase shifter section of $3.47 \mathrm{~mm} \times 3.17 \mathrm{~mm}$.

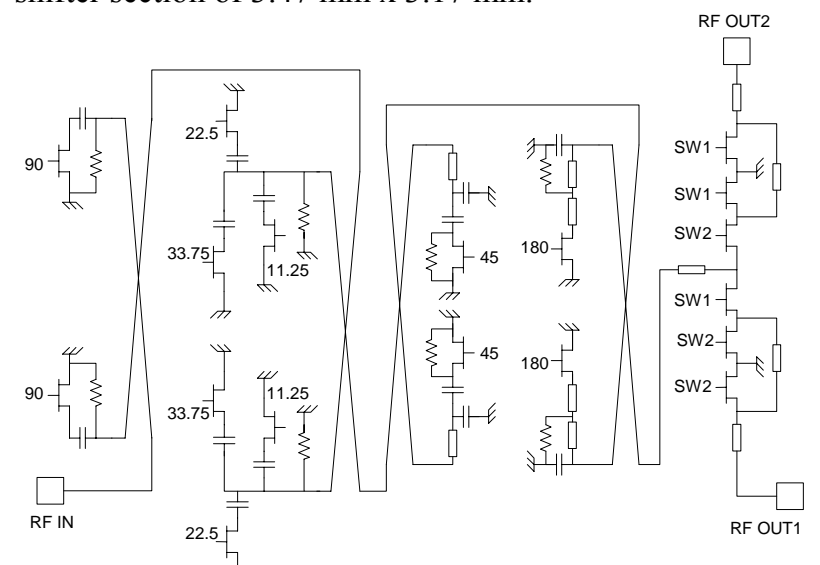

Figure 1. Schematic diagram of the proposed 5-bit phase shifter MMIC with SPDT switch. 


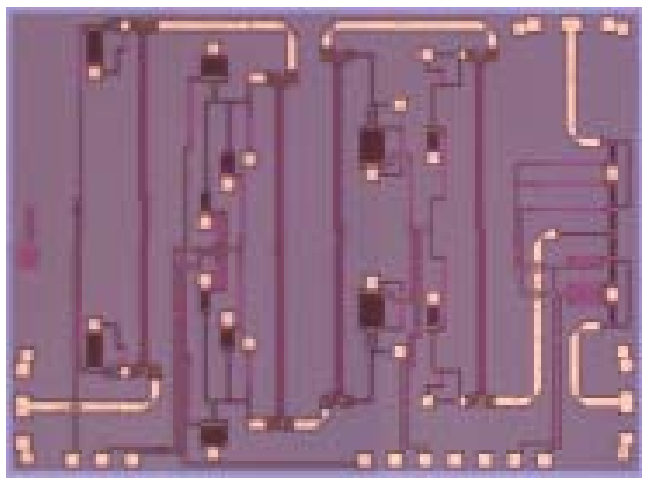

Figure 2. Photograph of the fabricated 5-bit phase shifter MMIC with SPDT switch.

The schematic diagram of the novel $180^{\circ}$ reflective terminating circuit is shown in Figure 3. It consists of only a few circuit elements, an inductor $\mathrm{L}$, a capacitor $\mathrm{C}$ and an FET. The FET is used as a switching element.

Figure 4 shows equivalent circuits of series and parallel LC states of the $180^{\circ}$ reflective terminating circuit. $\mathrm{R}_{\mathrm{on}}$ and $\mathrm{C}_{\text {off }}$ are the on-state resistance and the off-state capacitance of FET, respectively. When the FET turns on, the circuit corresponds to parallel $\mathrm{LC}$ circuit consisting of $\mathrm{L}$ and $\mathrm{C}$ shown in Figure 4 (a) by neglecting $\mathrm{R}_{\text {on }}$. When the FET is pinched off, the circuit can be simplified to a series $\mathrm{LC}$ circuit consisting of $\mathrm{L}$ and $\mathrm{C}_{\text {off }}$ shown in Figure 4 (b) if admittance of $\mathrm{C}$ is small enough to be neglected. Therefore, the circuit shown in Figure 3 can be regarded as a switching series/parallel LC circuit. When the resonant frequency of the series and the parallel LC circuits is set to be $\omega_{0}$, circuit elements of $\mathrm{L}, \mathrm{C}$ and $\mathrm{C}_{\text {off }}$ are determined uniquely given as follows

$$
\begin{gathered}
\mathrm{L}=\frac{\mathrm{Z}_{0}}{\omega_{0}} \\
\mathrm{C}=\mathrm{C}_{\text {off }}=\frac{1}{\omega_{0} \mathrm{Z}_{0}}
\end{gathered}
$$

$\mathrm{Z}_{0}$ is the impedance of the port of 3-dB Lange coupler. In the above condition, a phase difference between reflection phase in the series LC state and that in the parallel LC state is exactly $180^{\circ}$ for all frequencies theoretically. The theory was described in reference [8] in more detail. However, the return loss in the parallel LC state particularly appears at $\omega_{0}$ by the non-zero $R_{\text {on }}$. In practical design, $\omega_{0}$ has to be optimally determined higher than the operating frequency band. Also, a resistor is incorporated between source and drain terminals of FET to equalize the magnitude of reflection coefficient in series and parallel
LC states. That resistor has a negligible effect on phase difference and impedance of the circuit.

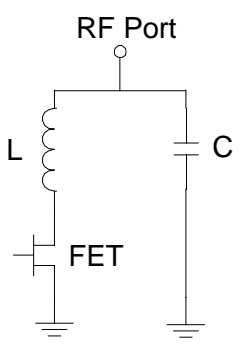

Figure 3. Schematic diagram of the novel $180^{\circ}$ reflective terminating circuit.

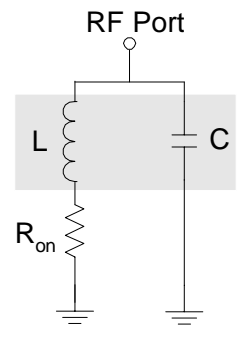

(a)

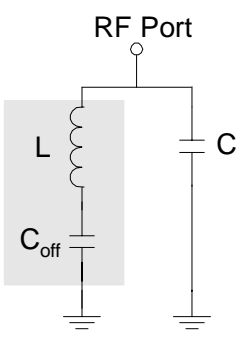

(b)
Figure 4. Equivalent circuits of the novel $180^{\circ}$ reflective terminating circuit. (a) Parallel LC State. (b) Series LC State.

The $90^{\circ}, 45^{\circ}$ and $22.5^{\circ} / 11.25^{\circ}$ bit reflective terminating circuits are based on digitally controlled capacitive circuit design. These circuits are composed of capacitors and switching FETs. The total capacitances of the reflective terminating circuit are set to be $\mathrm{C}_{\mathrm{a}}$ and $\mathrm{C}_{\mathrm{b}}$ in the phase shift state and the phase reference state, respectively. To obtain broad-band phase shift characteristics, $C_{a}$ and $C_{b}$ are determined as follows

$$
\mathrm{C}_{\mathrm{a}}=\frac{1}{\omega_{\mathrm{c}} \mathrm{Z}_{0}} \mathrm{e}^{\frac{\Delta \theta}{2}}, \quad \mathrm{C}_{\mathrm{b}}=\frac{1}{\omega_{\mathrm{c}} \mathrm{Z}_{0}} \mathrm{e}^{-\frac{\Delta \theta}{2}}
$$

$\omega_{\mathrm{c}}$ is the center frequency of the operating frequency band. $\Delta \theta$ is the desirable phase shift.

The $11.25^{\circ}$ and $22.5^{\circ}$ bit reflective terminating circuit are combined into one circuit which has four phase shift states. This circuit design can achieve size reduction and low loss characteristics compared with a bit circuit fabricated separately. To realize $33.75^{\circ}$ phase shift, aseries combination of a capacitor and a FET is added shown in Figure 1. 


\section{MEASUREMENT RESULTS}

The measured results of the fabricated 5-bit phase shifter MMIC with SPDT switch in all 32 phase states are shown in Figure 5-7 with a control voltage of $-3.0 \mathrm{~V}$. The measured input and output return loss were $8.8 \mathrm{~dB}$ and $11.8 \mathrm{~dB}$ at the worst case respectively in the 6 to $18 \mathrm{GHz}$ band. The typical insertion loss was $9.4 \mathrm{~dB}+/-1.4 \mathrm{~dB}$ over the same frequency range. A maximum RMS phase error was $7^{\circ}$ plotted in Figure 8, and a maximum RMS amplitude error was $0.33 \mathrm{~dB}$ plotted in Figure 9 in the operating band frequency. In particularly, the chip also showed very good performances over the typical midband. At $10 \mathrm{GHz}$, the insertion loss was $9 \mathrm{~dB}+/-0.16 \mathrm{~dB}$, and the RMS phase error was $2.6^{\circ}$. The 5-bit phase shifter with SPDT switch still keeps effective performance over the designed frequency band. The RMS phase error over high frequency band was mainly due to phase shift error of the $180^{\circ}$ bit circuit. Some improvements of the performance of the $180^{\circ}$ bit circuit are possible. The measured performance is summarized in Table 1 .

The measurements of the MMIC with a solo SPDT switch are shown in Figure 10. The measured insertion loss was less than $1.48 \mathrm{~dB}$ over the 6 to $18 \mathrm{GHz}$ band, and the isolation was more than $30.9 \mathrm{~dB}$ in the operating bandwidths.

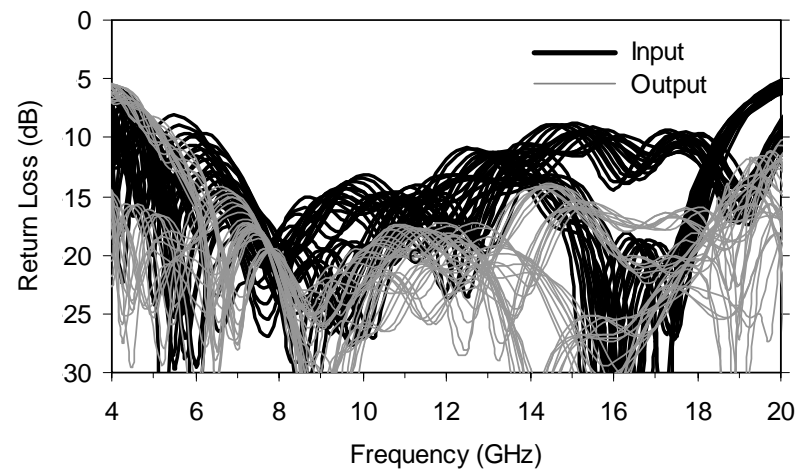

Figure 5. Measured input and output return loss in all 32 phase states.

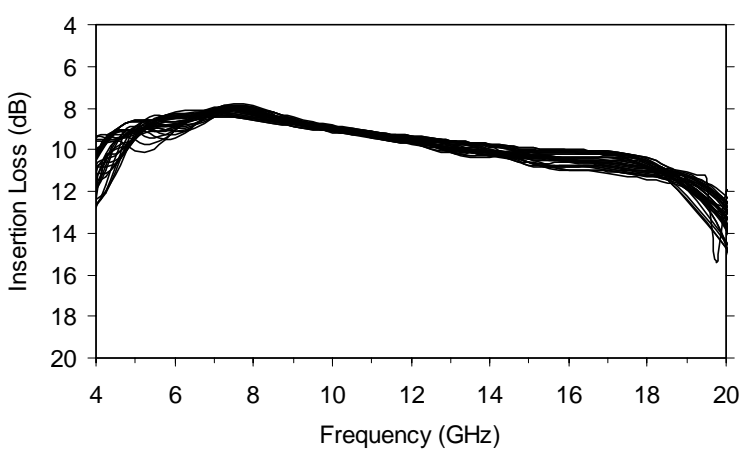

Figure 6. Measured insertion loss in all 32 phase states.

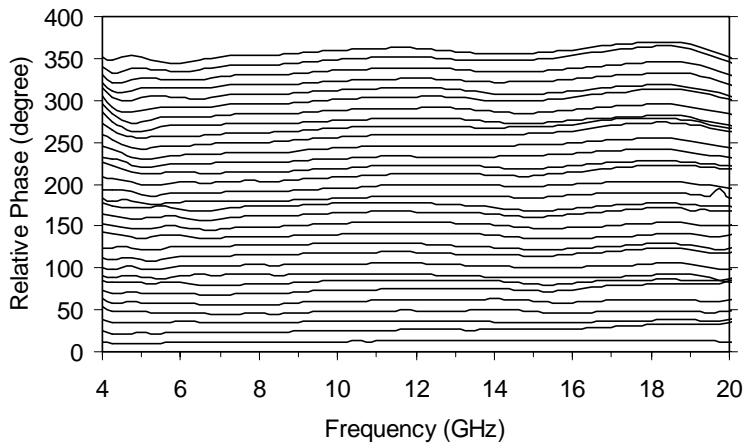

Figure 7. Measured phase shift in all 32 phase states.

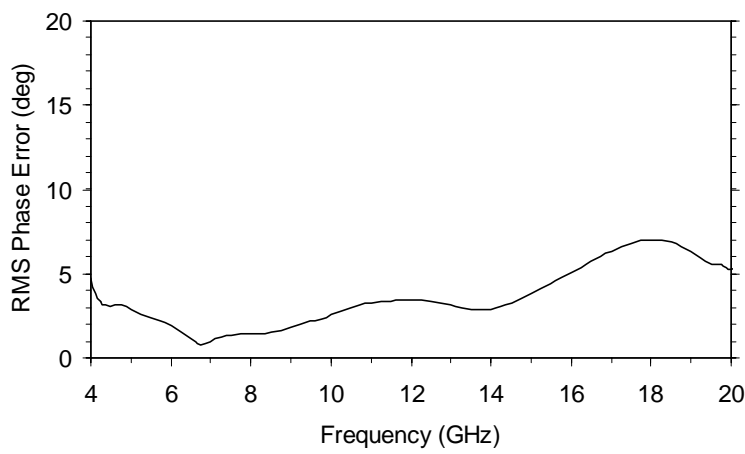

Figure 8. Measured RMS phase error. 


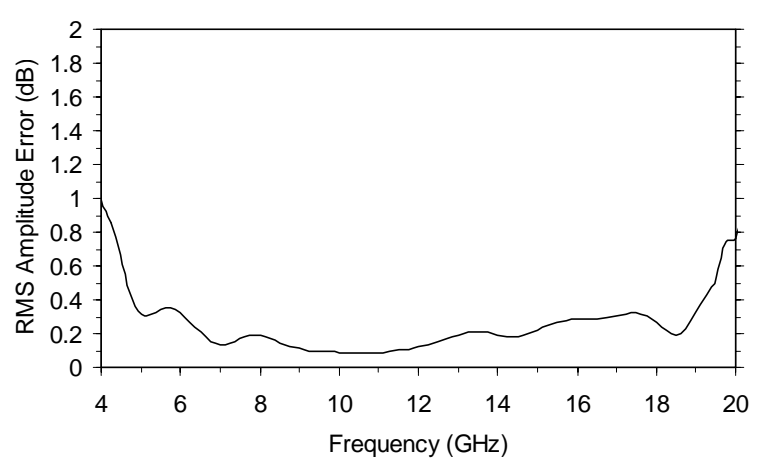

Figure 9. Measured RMS amplitude error.

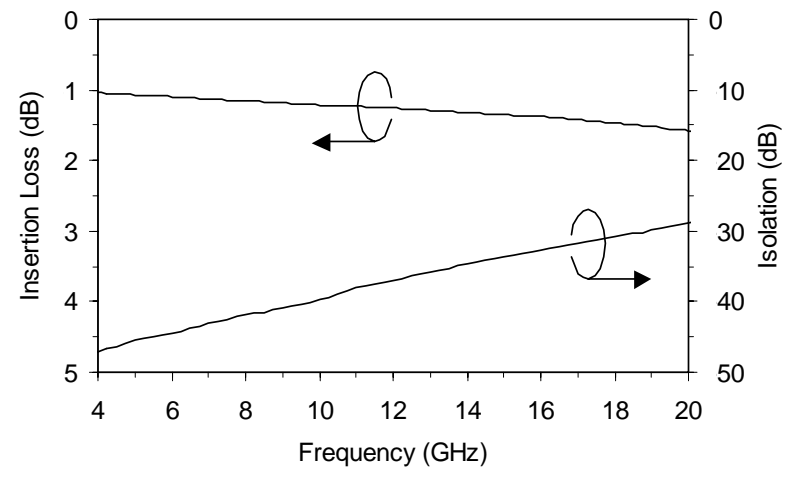

Figure 10. Measured insertion loss and isolation of the SPDT switch.

Table 1. Summary of the measured performance of the 5-bit phase shifter MMIC with SPDT switch over the 6 to $18 \mathrm{GHz}$.

\begin{tabular}{|l|r|}
\hline Parameter & Value \\
\hline \hline Min. Input Return Loss & $8.8 \mathrm{~dB}$ \\
\hline Average Input Return Loss & $12.2 \mathrm{~dB}$ \\
\hline Min. Output Return Loss & $11.8 \mathrm{~dB}$ \\
\hline Average Ouput Return Loss & $16.9 \mathrm{~dB}$ \\
\hline Average Insertion Loss & $7^{\circ}$ \\
\hline Max. RMS Phase Error & $3.3^{\circ}$ \\
\hline Average RMS Phase Error & $0.33 \mathrm{~dB}$ \\
\hline Max. RMS Amplitude Error & $0.19 \mathrm{~dB}$ \\
\hline Average RMS Amplitude Error & \\
\hline
\end{tabular}

\section{CONCLUSION}

A broad-band 5-bit reflection type phase shifter was developed. The phase shifter employs a novel ultra broadband circuit design technique utilizing a series/parallel LC circuit for a $180^{\circ}$ phase shift over all frequencies. The fabricated phase shifter MMIC with SPDT switch suitable for $\mathrm{T} / \mathrm{R}$ modules has successfully demonstrated a typical insertion loss of $9.4 \mathrm{~dB}+/-1.4 \mathrm{~dB}$, a maximum RMS amplitude error of $0.33 \mathrm{~dB}$ and a maximum RMS phase error of $7^{\circ}$ over the 6 to $18 \mathrm{GHz}$ band. That excellent broad-band performance is suitable for wideband phased array applications.

\section{REFERENCES}

[1] R. V. Garver, "Broad-Band Diode Phase Shifters," IEEE Trans. On Microwave Theory and Techniques, Vol. MTT20, No. 5, pp. 314-323, May 1972.

[2] D. C. Boire, G. St. Onge, C. Barratt, G.B. Norris and A. Moysenko, "4:1 Bandwidth Digital Five Bit MMIC Phase Shifters," IEEE Microwave and Millimeter-Wave Monolithic Circuits Symposium, pp.69-73, 1989.

[3] T. A. Murphy, J. W. Gipprich, M. E. Hines and D. Kryger, "A Six-Bit GMIC Phase Shifter for 6-18 GHz," IEEE International Microwave Symposium Dig., pp. 1171-1174, 1992.

[4] M. Schindler, Y. Ayasli, A. Morris and L. Hanes, "Monolithic 6-18 GHz 3-bit Phase Shifter," IEEE GaAs IC Symposium, pp. 129-132, 1985.

[5] K. M. Simon, M. J. Schindler, V. A. Mieczkowski, P. F. Newman, M. E. Goldfarb, E. Reese and B. A. Small, "A Production Ready, 6-18 GHz Five-Bit Phase Shifter with Integrated CMOS Compatible Digital Interface Circuitry," IEEE GaAs IC Symposium, pp.251-254, 1991.

[6] D. C. Boire, J. E. Dejenford and M. Cohn, "A 4.5 to 18 $\mathrm{GHz}$ Phase Shifter," IEEE International Microwave Symposium Dig., pp. 601-604, 1985.

[7] D. M. Krafcsik, S. A. Imhoff, D. E. Dawson and A. L. Conti, "A Dual-Varactor Analog Phase Shifter Operating at 6 to $18 \mathrm{GHz}$," IEEE Trans. On Microwave Theory and Techniques, Vol. 36, No. 12, pp. 1938-1941, Dec. 1988.

[8] K. Miyaguchi, M. Hieda, K. Nakahara, H. Kurusu, M. Nii, M. Kasahara, T. Takagi and S. Urasaki, "An Ultra-BroadBand Reflection-Type Phase-Shifter MMIC With Series and Parallel LC Circuits," IEEE Trans. On Microwave Theory and Techniques, Vol. 49, No. 12, pp.2446-2452, Dec. 2001. 